\title{
Differential Accumulation of Aroma Compounds in Normal Green and Albino-Induced Yellow Tea (Camellia sinensis) Leaves
}

\author{
Fang Dong ${ }^{1,+}$, Lanting Zeng ${ }^{2,3,+}$, Zhenming $\mathrm{Yu}^{2,3}$, Jianlong $\mathrm{Li}^{4}$, Jinchi Tang ${ }^{4}$, Xinguo Su ${ }^{1, *}$ \\ and Ziyin Yang 2,3,*(D) \\ 1 Guangdong Food and Drug Vocational College, Longdongbei Road 321, Tianhe District, \\ Guangzhou 510520, China; dongfangxyz@163.com \\ 2 Key Laboratory of South China Agricultural Plant Molecular Analysis and Genetic Improvement \& \\ Guangdong Provincial Key Laboratory of Applied Botany, South China Botanical Garden, \\ Chinese Academy of Sciences, Xingke Road 723, Tianhe District, Guangzhou 510650, China; \\ zenglanting@scbg.ac.cn (L.Z.); zhenming311@scbg.ac.cn (Z.Y.) \\ 3 College of Advanced Agricultural Sciences, University of Chinese Academy of Sciences, \\ No.19A Yuquan Road, Beijing 100049, China \\ 4 Tea Research Institute, Guangdong Academy of Agricultural Sciences \& Guangdong Provincial Key \\ Laboratory of Tea Plant Resources Innovation and Utilization, Dafeng Road 6, Tianhe District, \\ Guangzhou 510640, China; skylong.41@163.com (J.L.); tangjinchi@126.com (J.T.) \\ * Correspondence: suxg@gdyzy.edu.cn (X.S.); zyyang@scbg.ac.cn (Z.Y.); \\ Tel.: +86-20-2885-4755 (X.S.); +86-20-3807-2989 (Z.Y.); Fax: +86-20-3807-2989 (Z.Y.) \\ + These authors contributed equally to this work.
}

Received: 26 September 2018; Accepted: 17 October 2018; Published: 18 October 2018

\begin{abstract}
Tea (Camellia sinensis) cultivars with green leaves are the most widely used for making tea. Recently, tea mutants with white or yellow young shoots have attracted increasing interest as raw materials for making "high-quality" tea products. Albino teas are generallycharacterized as having metabolites of relatively high amino acid content and lower catechin content. However, little is known about aroma compounds in albino tea leaves. Herein, we compared original normal leaves (green) and light-sensitive albino leaves (yellow) of cv. Yinghong No. 9. GC-MS was employed to analyze endogenous tea aroma compounds and related precursors. Quantitative real time PCR was used to measure expression levels of genes involved in biosyntheses of tea aromas. The total contents of most endogenous free tea aromas, including aroma fatty acid derivatives, aroma terpenes, and aroma phenylpropanoids/benzenoids, and their glycosidically bound aroma compounds, were lower in yellow leaves than in green leaves. The content of the key precursor geranyl diphosphate (GDP) and expression levels of key synthetic genes involved in the formation of linalool, a major aroma compound in cv. Yinghong No. 9, were investigated. Linalool content was lower in albino-induced yellow leaves, which was due to the lower GDP content compared with normal green leaves.
\end{abstract}

Keywords: albino; aroma; Camellia sinensis; geranyl diphosphate; light-sensitive; linalool; tea

\section{Introduction}

The drink made from tea (Camellia sinensis) leaves is the second most popular beverage worldwide after water. This popularity is attributed to tea leaves having health benefits and containing flavor related metabolites, such as polyphenols, caffeine, amino acids, aroma compounds, vitamins, and carbohydrates [1-3]. These metabolites can be affected by preharvest agronomy management and postharvest manufacturing processes [3-5]. Furthermore, these metabolites have different distributions 
in different tea varieties. In general, tea cultivars with green leaves are the most widely used for making tea. In recent years, tea mutants with white or yellow young shoots have attracted increasing interest from tea researchers and tea manufacturers as potential raw materials for making "high-quality" tea products. According to their different responses to the environment, albino teas can be divided into ecologically sensitive types (including light-sensitive and low-temperature-sensitive types), in which leaf colors change to white or yellow under certain environmental conditions, or ecologically sensitive types where the leaf color is permanently white or yellow regardless of the environment $[6,7]$. Temperature-sensitive mutants grow white or yellow shoots below certain temperatures, such as $20{ }^{\circ} \mathrm{C}$, during early spring, with examples including 'Xiaoxueya' and 'White leaf No. $1^{\prime}$ ' cultivars [8,9]. Light-sensitive mutants grow yellow shoots under strong light illumination, with examples including 'Jinguang', 'Yu-Jin-Xiang', and 'Huangjinya' cultivars [10-12]. These albino tea leaves have white or yellow shoots owing to chlorophyll deficiencies and, in terms of metabolite characteristics, generally have relatively high amino acid contents and lower catechin contents [6,7]. However, little is known about the occurrence of aroma compounds in albino tea leaves. Although no reported reference is available, most tea products made from albino-induced yellow leaves generally showed weak aroma properties according to information from tea manufacturers. From the viewpoint of plants, the albino tea leaves have defects in the chloroplasts [13], which can affect the biosynthesis of specialized metabolites such as catechins, which are lower in content in albino-induced yellow tea leaves [6,7]. Accumulation of proteinaceous amino acids in albino-induced yellow tea leaves possibly results from degradation of chloroplast-located proteins. Higher content of some non-proteinaceous amino acid such as L-theanine in albino tea leaves is due to weak catabolism of the amino acid in albino tea leaves [13]. The objective of this study is to investigate whether albinism affects tea aroma profiles and if it is a positive or negative influence. Aroma is an important factor affecting the character and quality of tea $[4,5]$. In general, phenolic compounds and amino acids account for $18-36 \%$ and $1-4 \%$ of tea content, respectively, while aroma compounds account for less than $0.03 \%$ [3]. Therefore, improving tea aroma quality is of significant interest and has attracted increasing research attention. Understanding the biosyntheses and regulation mechanisms of tea aroma compounds is key to safely and effectively improving tea quality [5]. In fresh tea leaves with living leaf cells, tea aroma compounds are mainly classified into aroma fatty acid derivatives (VFADs), aroma terpenes (VTs), and aroma phenylpropanoids/benzenoids (VPBs) according to the different biosynthetic pathways, and similar to other plants $[4,5]$. In fresh tea leaves, not many aroma compounds are detected, and they occur conservatively in most tea cultivars. During the tea manufacturing process, many aroma compounds, which may involve some new compounds, are produced $[4,5]$.

In this study, to exclude the effects of genetic background, geography, and climate, C. sinensis cv. Yinghong No. 9 original cultivar (green) and its light-sensitive mutant (albino-induced yellow), which shares the same genetic background were sampled in pairs under the same growth conditions. We compared the aroma profiles (VFADs, VTs, and VPBs), major aroma compound related key precursor metabolite levels, and key synthetic gene expressions, of normal green tea leaves and albino-induced yellow tea leaves. This information will advance our understanding of the profiles of tea aromas and their formation in albino tea leaves.

\section{Results}

\subsection{Comparisons of Endogenous Free Aroma Compound Contents between Albino-Induced Yellow Leaves and Normal Green Leaves}

Figure S1 (supplementary information) shows GC-MS TIC chromatograms of endogenous free aroma compounds in normal green and albino-induced yellow tea leavescollected in April, August, and December. Both normal green tea leaves and albino-induced yellow leaves contained the three major classes of aromas, namely VFADs (Figure 1A), VTs (Figure 1B), and VPBs (Figure 1C). In the spring tea leaves collected in April, although not all of the content of the aroma compounds were lower in the albino-induced yellow leaves than in the normal green leaves, the content of the two 
most abundant free aroma compounds, (Z)-3-hexenol and linalool (Figure 1A,B), was significantly less, which led to a lower total content of the detected aromas in albino-induced yellow leaves than in normal green leaves (Figure 1D). To investigate whether different seasons affected the endogenous free aroma compounds in albino-induced yellow leaves and normal green leaves, we compared the aroma profiles of both types of tea leaves picked in August (summer-autumn tea) (Figure 2) and December (winter tea) (Figure 3). In the tea samples picked in August and December, the total content of the detected aromas was lower in the albino-induced yellow leaves than in the normal green leaves (Figures 2D and 3D), which showed similar trends to those in the spring tea (Figure 1D). In addition, endogenous free aroma compound contents were affected by tea seasons. The aroma compounds of spring tea and winter tea were generally higher than those of summer-autumn tea (Figures 1-3).
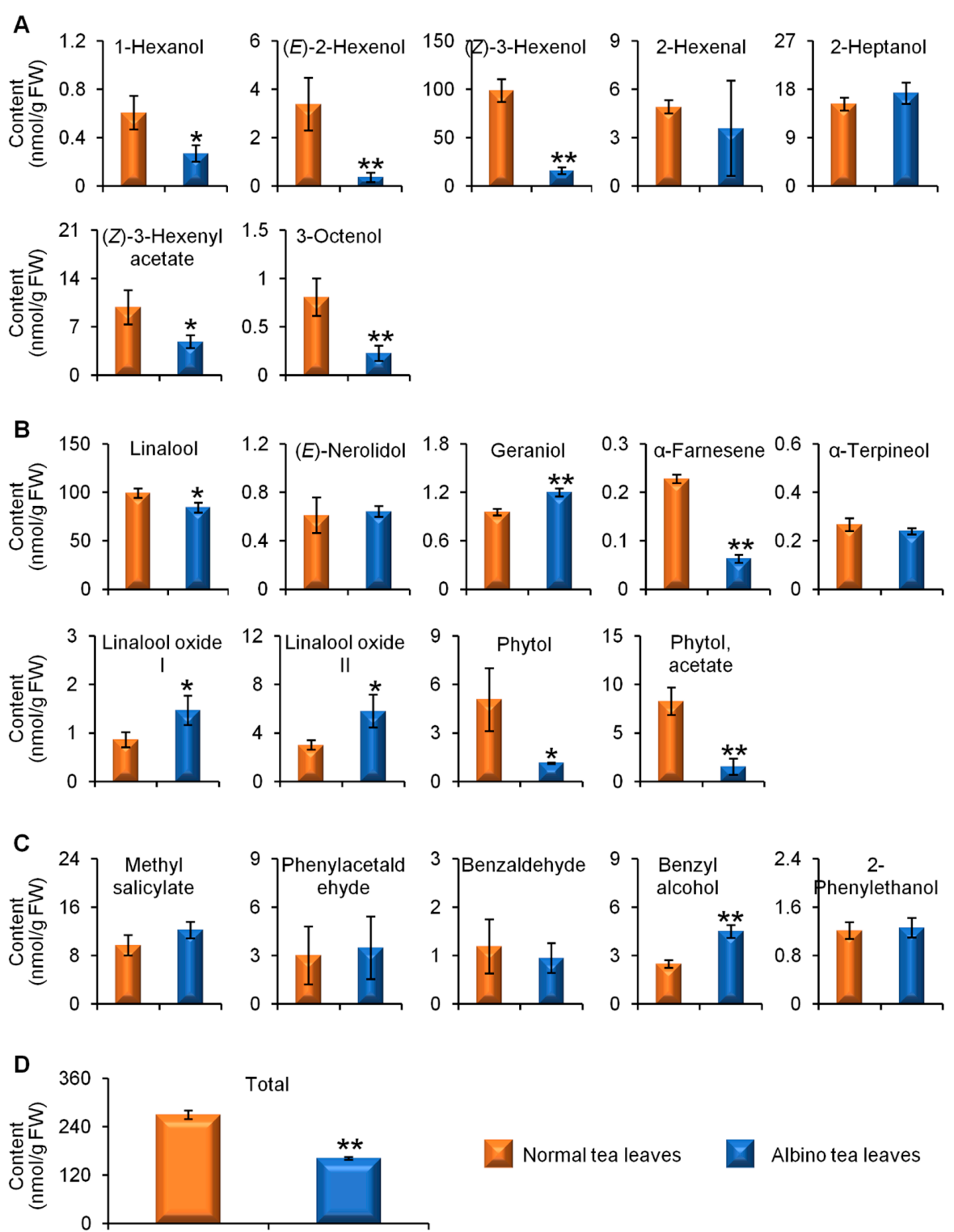

Figure 1. Differential accumulation of endogenous free aroma compounds in normal green and albino-induced yellow tea (Camellia sinensis) leaves (cv. Yinghong No. 9) collected in April. (A) aroma fatty acid derivatives; (B) aroma terpenes; (C) aroma phenylpropanoids/benzenoids; (D) total content of aroma compounds shown in (A-C). The identification and quantitative analysis of the aroma compounds is summarized in Table S1 (Supplementary Information). All data are expressed as mean \pm S.D. $(n=3)$. Significant differences between normal and albino tea leaves are indicated $\left({ }^{*} p \leq 0.05\right.$, and $\left.{ }^{* *} p \leq 0.01\right)$. FW, fresh weight. 

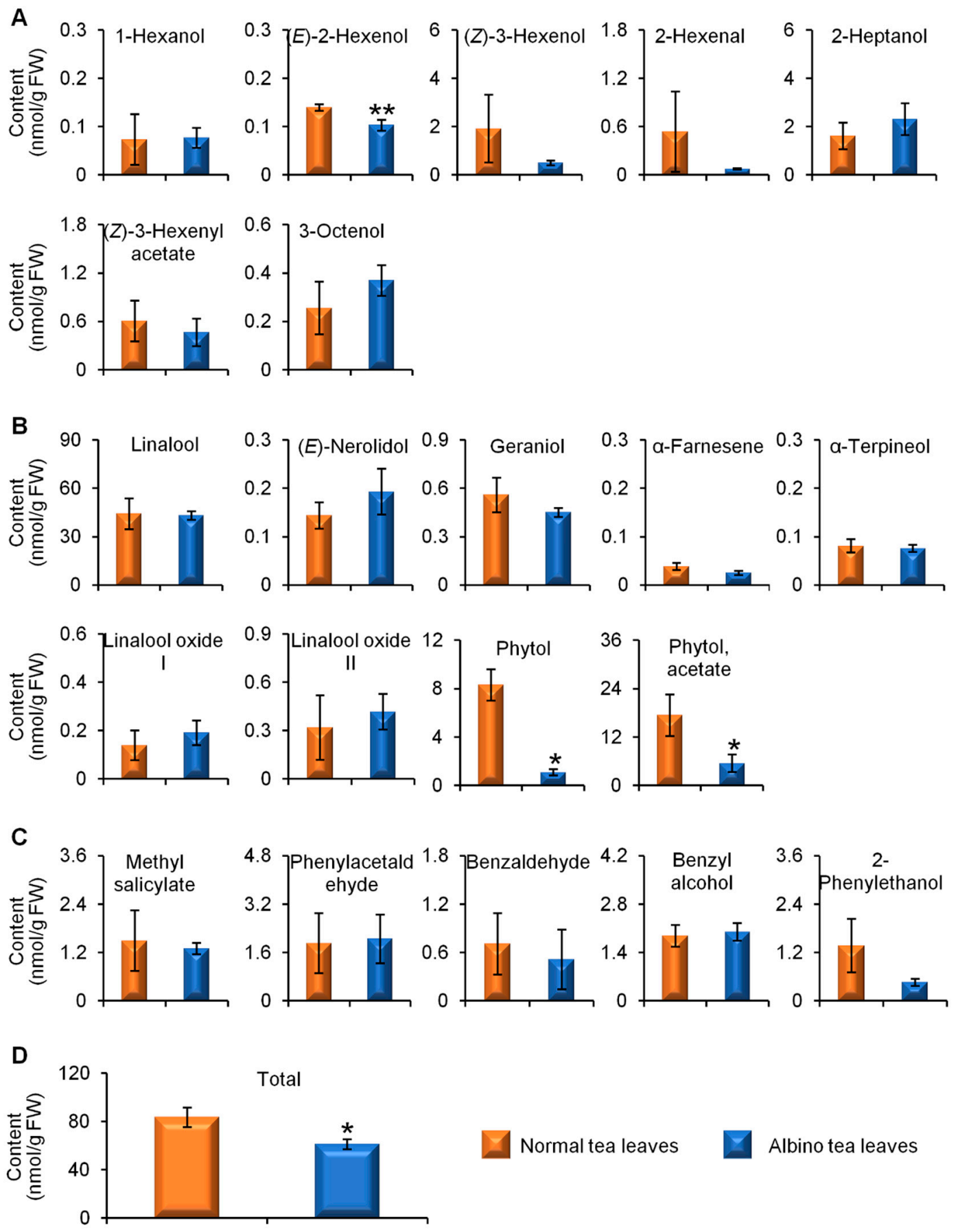

Normal tea leaves Albino tea leaves

Figure 2. Differential accumulation of aroma compounds in normal green and albino-induced yellow tea (Camellia sinensis) leaves (cv. Yinghong No. 9) collected in August. (A) aroma fatty acid derivatives; (B) aroma terpenes; (C) aroma phenylpropanoids/benzenoids; (D) total content of aroma compounds shown in (A-C). The identification and quantitative analysis of the aroma compounds is summarized in Table S1 (Supplementary Information). All data are expressed as mean \pm S.D. $(n=3)$. Significant differences between normal and albino tea leaves are indicated $\left({ }^{*} p \leq 0.05\right.$, and $\left.{ }^{* *} p \leq 0.01\right)$. FW, fresh weight. 

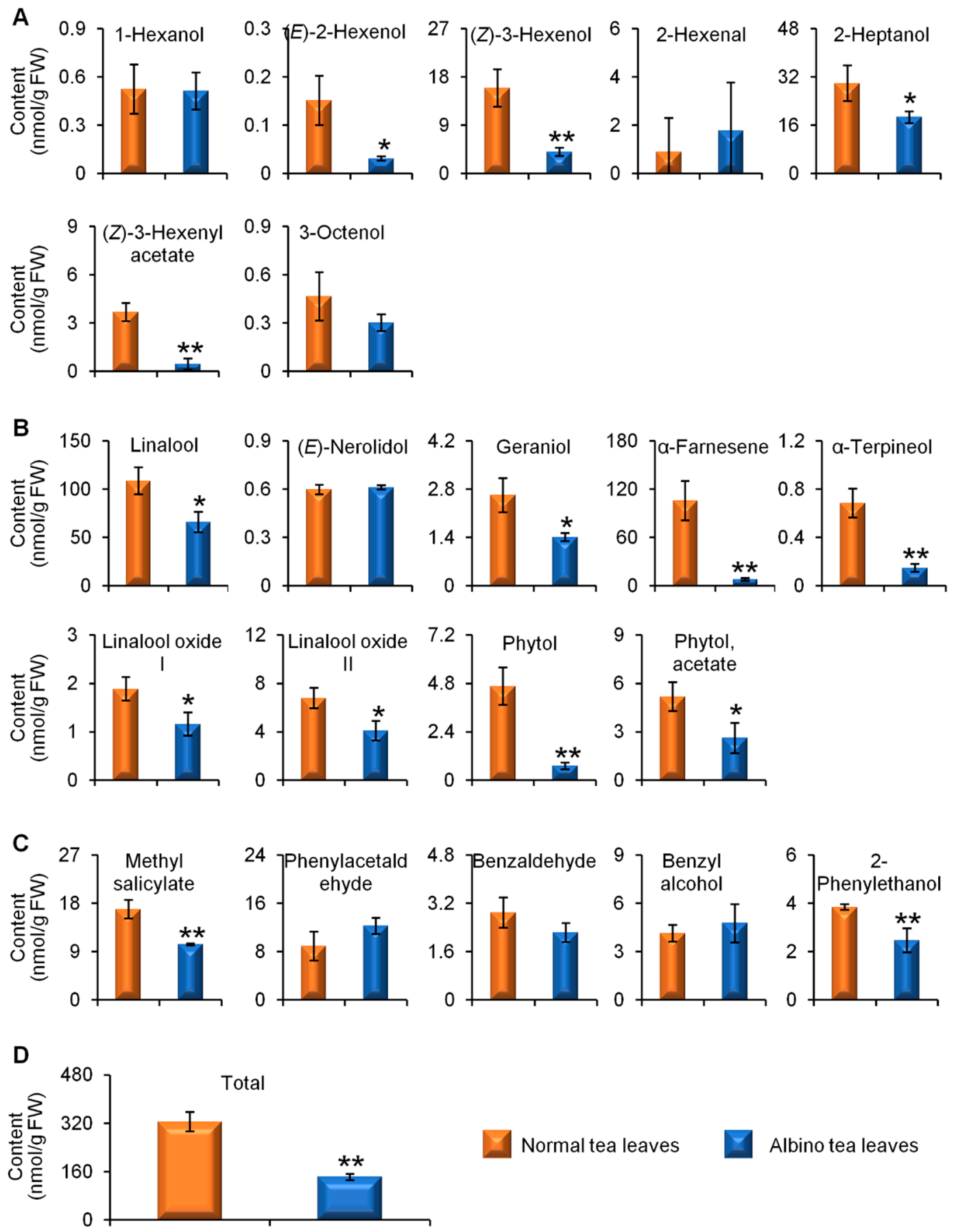

Normal tea leaves

Albino tea leaves

Figure 3. Differential accumulation of aroma compounds in normal green and albino-induced yellow tea (Camellia sinensis) leaves (cv. Yinghong No. 9) collected in December. (A) aroma fatty acid derivatives; (B) aroma terpenes; (C) aroma phenylpropanoids/benzenoids; (D) total content of aroma compounds shown in $(\mathbf{A}-\mathbf{C})$. The identification and quantitative analysis of the aroma compounds is summarized in Table S1 (Supplementary Information). All data are expressed as mean \pm S.D. $(n=3)$. Significant differences between normal and albino tea leaves are indicated $\left({ }^{*} p \leq 0.05\right.$, and ${ }^{* *} p \leq 0.01$ ). FW, fresh weight.

2.2. Comparison of Glycosidically Bound Aroma Compound Contents and Related Gene Expression Levels between Albino-Induced Yellow Leaves and Normal Green Leaves

In addition to their free forms, aroma compounds can occur in plants as glycosidically bound forms that are more water soluble and less reactive than their free aglycone counterparts. We also 
compared glycosidically bound aroma compounds in albino-induced yellow leaves and normal green leaves. The results showed that the content of most glycosidically bound aroma compounds was lower in albino-induced yellow leaves than in normal green leaves (Figure 4A-D). Furthermore, the expression levels of glycosidically conjugated aroma compound formation and hydrolysis-related genes ( $\beta$-primeverosidase (PD), glycosyltransferases (GT1 and GT2)) showed no significant differences between albino-induced yellow leaves and normal green leaves (Figure $4 \mathrm{E}$ ).
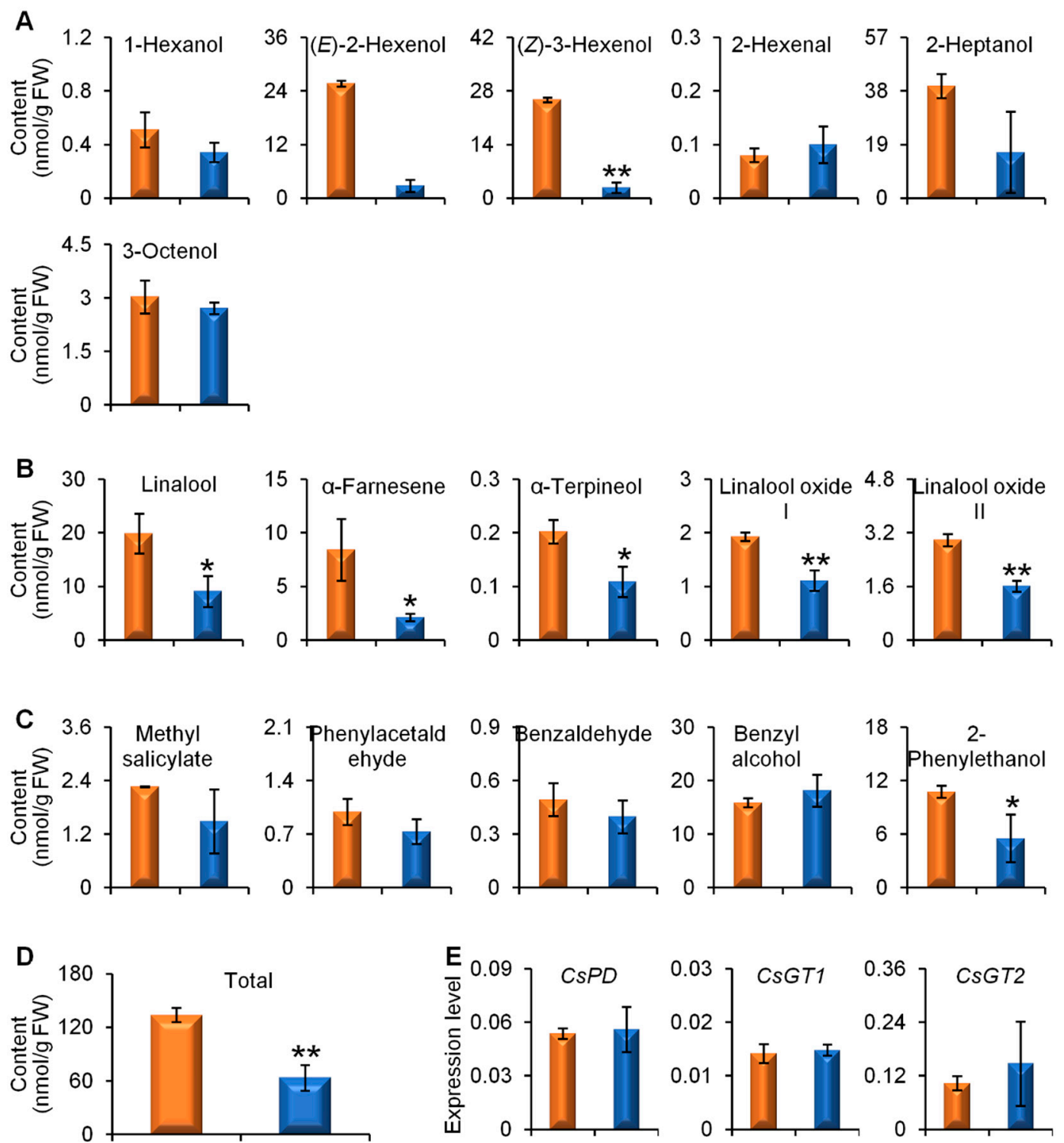

Normal tea leaves

Albino tea leaves

Figure 4. Differential accumulation of glycosidically bound aromas (GBVs) (A-D) and expression levels of genes involved in aroma compounds synthesis from glycosidic hydrolysis (E) in normal green and albino-induced yellow tea (Camellia sinensis) leaves (cv. Yinghong No. 9) collected in April. The content of GBVs were calculated based on the enzyme reaction products under $\beta$-primeverosidase and $\beta$-glucosidase catalyzation. (A) aroma fatty acid derivatives; (B) aroma terpenes; (C) aroma phenylpropanoids/benzenoids; (D) total content of aroma compounds shown in (A-C). The identification and quantitative analysis of the aroma compounds is summarized in Table S1 (Supplementary Information). FW, fresh weight. (E) PD, $\beta$-primeverosidase; GT, glycosyltransferase. All data are expressed as mean \pm S.D. $(n=3)$. Significant differences between normal and albino tea leaves are indicated $\left(* p \leq 0.05\right.$, and $\left.{ }^{* *} p \leq 0.01\right)$. 
2.3. Comparison of Linalool Synthase Gene Expression Levels and GDP Content between Albino-Induced Yellow Tea Leaves and Normal Green Tea Leaves

In the present study, linalool is a major endogenous free aroma compound in cv. Yinghong No. 9 tea leaves (Figures 1-3). Therefore, we investigated the reason for the lower linalool content in albino-induced yellow tea leaves. The four linalool synthase genes involved in linalool formation from GDP, namely, CsLIS1, CsLIS2, CsLIS/NES-1, and CsLIS/NES-2, showed no significant differences between the albino-induced yellow leaves and normal green leaves (Figure 5A). However, the GDP content in albino-induced yellow leaves was lower than that in normal green leaves (Figure 5B), which might be a reason for the lower amount of linalool in albino-induced yellow tea leaves.

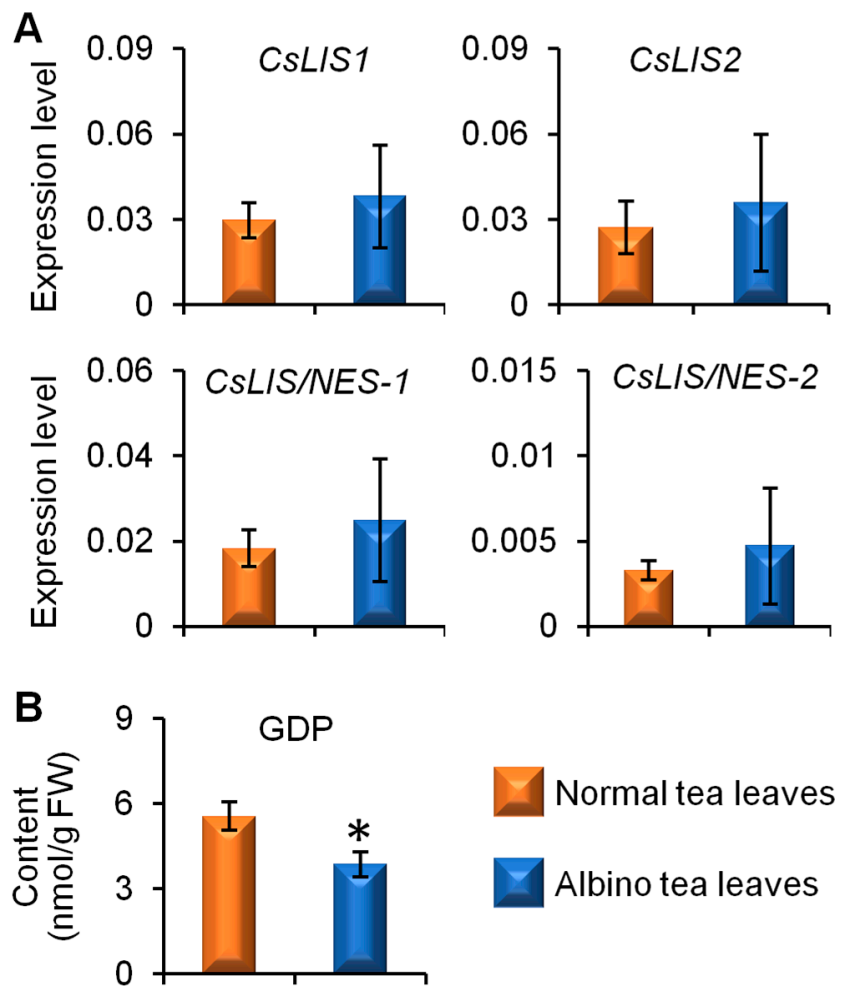

Figure 5. Differential expression levels of genes involved in linalool synthesis (A) and accumulation of geranyl diphosphate (GDP) (B) in normal green and albino-induced yellow tea (Camellia sinensis) leaves (cv. Yinghong No. 9) collected in April. (A) LIS, linalool synthase; LIS/NES, linalool synthase/(E)-nerolidol synthase. (B) FW, fresh weight. All data are expressed as mean \pm S.D. $(n=3)$. Significant differences between normal and albino tea leaves are indicated $\left({ }^{*} p \leq 0.05\right)$.

\section{Discussion}

Obviously, albino tea leaves are yellow due to a lack of chlorophyll compared with the original green cultivars, which leads to defects in the chloroplast ultrastructure and composition, and a reduction in the number of chloroplasts. In contrast, the original green cultivars have normal chloroplast grana, stroma, thylakoids, and starch grains [8]. The albinism mechanism in albino tea cultivars and changes in non-aroma metabolites during leaf development or shade treatment have been studied using proteomics, transcriptomics, and metabolomics analysis [10-14]. In previous studies, the tea samples selected have different genetic backgrounds, and different geographic or climate characteristics, hence, these factors may interfere with results concerning the effects of albinism on metabolites in tea leaves. In our previous study, C. sinensis cv. Yinghong No. 9 original cultivar (green) and its light-sensitive mutant (albino-induced yellow), which shares the same genetic background, were used in pairs under the same growth conditions to investigate the differential accumulation of specialized metabolite L-theanine in green and albino-induced yellow tea leaves [13]. Although 
the availability of ethylamine (a biosynthetic precursor of L-theanine) is crucial for the differential accumulation of L-theanine between tea and other plants [15], weak L-theanine catabolism was responsible for the higher L-theanine content in albino-induced yellow leaves compared with normal green leaves [13]. The relatively low catechin contents in the albino tea leaves might also be related to the limited catechin biosynthesis in albino tea leaves [11,12]. Complete dark treatment of normal green tea leaves led to etiolated leaves, which had the same appearance as albino-induced yellow leaves [16]. The dark treatment significantly increased the free amino acid content and decreased the soluble protein content in tea leaves. Chloroplast numbers were lower in dark-treated leaves and the soluble protein content in chloroplasts isolated from dark-treated leaves was lower compared with the control, suggesting that proteolysis of the chloroplast proteins contributed to amino acid accumulation in dark-treated leaves [17]. The accumulation of proteinaceous amino acids possibly resulted from the degradation of chloroplast-located proteins. Previous reports have mostly focused on the effects of albinism on non-aroma metabolites in tea leaves. In the present study, the amount of major endogenous free tea aroma compounds (aroma metabolites) was lower in yellow leaves than in green leaves (Figures 1-3). Furthermore, the content of most glycosidically bound aroma compounds was lower in the albino-induced yellow leaves than in the normal green leaves (Figure 4).

Although a genetic transformation system has yet to be established for tea, in the last decade, most identified genes involved in the formation of tea aroma compounds have been expressed using Escherichia coli, yeast, and insect cells, or in transient overexpression model plant systems and functionally characterized in vitro [5]. $\beta$-Glycosidases, including CsPD, CsGlus, and CsGTs, involved in the transformation of glycoside-bound aromas and free aromas in tea leaves have been intensively studied and functionally characterized [18-22]. Furthermore, several enzymes and genes involved in the final steps of the biosynthesis of tea aroma compounds, such as CsTPSs for (S)-linalool and (E)-nerolidol formation, and CsTSA and CsTSBs for indole formation, have been isolated, identified, and functionally characterized [23-26]. Some enzymes and genes involved in upstream pathways responsible for the formation of tea aroma compounds, such as CsLOXs for jasmine lactone formation and CsAAATs for benzaldehyde, benzyl alcohol, and methyl benzoate formation, have also been functionally characterized $[27,28]$. In the present study, the levels of genes involved in tea aroma formation, including CsPD, CsGT1, GsGT2, CsLIS1, CsLIS2, CsLIS/NES-1, and CsLIS/NES-2, were investigated to compare differences in the tea aroma biosynthetic abilities of albino-induced yellow leaves and normal green tea leaves (Figures $4 \mathrm{E}$ and $5 \mathrm{~A}$ ). As the functions of most genes involved in tea aroma formation investigated in the present study have been validated, the differences in metabolic flux between albino-induced yellow leaves and normal green tea leaves were more reliable. These investigated genes showed no significant difference between albino-induced yellow leaves and normal green leaves. However, the content of some key precursors of aroma compounds, such as GDP, was lower in albino-induced yellow leaves (Figure 5B).

\section{Materials and Methods}

\subsection{Chemicals and Regents}

n-Alkanes (C8-C40) were purchased from J\&K Scientific, Beijing, China. Benzaldehyde, benzyl alcohol, (Z)-3-hexenyl acetate, methyl salicylate, and 2-phenylethanol were purchased from Wako Pure Chemical Industries Ltd, Osaka, Japan. Ethyl n-decanoate, geraniol, 1-hexanol, (Z)-3-hexenol, phenylacetaldehyde, $\alpha$-farnesene, $(E)$-nerolidol, linalool, linalool oxide, polyvinylpolypyrrolidone (PVPP), and XAD-2 were purchased from Sigma-Aldrich Company Ltd., Louis, MO, USA. Geraniol was purchased from TCI Development Co., Ltd, Shanghai, China. The Quick RNA isolation kit was purchased from Huayueyang Biotechnology Co., Ltd., Beijing, China. The $2 \times$ SYBR Green Universal PCR Mastermix was purchased from Bio-Rad Laboratories, Richmond, CA, USA. 


\subsection{Plant Materials}

Three or four leaves of C. sinensis cv. Yinghong No. 9 and its yellow mutant (a light-sensitive variant) were used in the present study. C. sinensis cv. Yinghong No. 9 yellow mutant (a light-sensitive variant) and its original cultivar were picked at the Tea Research Institute, Guangdong Academy of Agricultural Sciences (Yingde, Guangdong, China), in April, August, and December.

\subsection{Extraction and Analysis of Endogenous Aroma Compounds in Tea Leaves}

The method used for extraction and analysis of endogenous aroma compounds in tea leaves was taken from our previous study with some modifications [29]. Finely powdered sample (1 g, fresh weight) was extracted with dichloromethane $(2.7 \mathrm{~mL})$ containing ethyl decanoate $(5 \mathrm{nmol})$ as an internal standardusing a shakerat room temperature overnight. The extraction solution was collected, dried using anhydrous sodium sulfate, and concentrated to $200 \mu \mathrm{L}$ under a stream of nitrogen. The extract $(1 \mu \mathrm{L})$ was then subjected to GC-MS analysis conforming on a GC-MS QP2010 SE (Shimadzu Corporation, Kyoto, Japan) equipped with GC-MS Solution software (Version 2.72, Shimadzu Corporation, Kyoto, Japan). Samples were injected into the GC injection port held at $230{ }^{\circ} \mathrm{C}$ for $1 \mathrm{~min}$, with all injections made in splitless mode. Aroma compounds were separated on a SUPELCOWAX 10 column (30 $\mathrm{m} \times 0.25 \mathrm{~mm} \times 0.25 \mu \mathrm{m}$, Supelco Inc., Bellefonte, PA, USA). Helium was used as the carrier gas, with a velocity of $1 \mathrm{~mL} / \mathrm{min}$. The initial GC oven temperature was $60{ }^{\circ} \mathrm{C}$ for $3 \mathrm{~min}$, which was ramped up to $240^{\circ} \mathrm{C}$ at a rate of $4{ }^{\circ} \mathrm{C} / \mathrm{min}$, and then held at $240{ }^{\circ} \mathrm{C}$ for $20 \mathrm{~min}$. Mass spectrometry (Shimadzu Corporation, Kyoto, Japan) was operated in full scan mode (mass range, $m / z$ 40-200).

The identification and quantitative analysis of the aroma compounds is summarized in Table S1 (Supplementary Information). 1-Hexanol, (Z)-3-hexenol, (Z)-3-hexenyl acetate, linalool, (E)-nerolidol, geraniol, $\alpha$-farnesene, linalool oxide, methyl salicylate, phenylacetaldehyde, benzaldehyde, benzyl alcohol, and 2-phenylethanol were identified by direct comparison with authentic standards. The quantitative analyses of these compounds were based on calibration curves, which were constructed by plotting the concentration of each compound against the peak area of the authentic standard. Some aroma compounds, for which no authentic standards were available in our lab were identified by comparison with retention indices (RI). The RIs of the compounds were calculated from an $n$-alkane series (C8-C40) [30]. Compounds with minor differences (less than 20) between the experimental RI and RI values cited in the literature were confirmed as common components. The relative content of each compound was calculated by comparison with the peak area of ethyl n-decanoate (internal standard). Some aroma compounds for which no standards and reference RI values were available, were identified by comparison with mass spectra, and these compounds were tentatively identified. In addition, the quantitative analysis of these compounds was the same as the one identified based on the reference RI value.

\subsection{Extraction and Analysis of GBVs in Tea Leaves}

The methods used for extraction and analysis of GBVs in tea leaves were according to the former study [22]. Five hundred $\mathrm{mg}$ (fresh weight) of finely powdered samples was extracted with $2 \mathrm{~mL}$ pre-cooling methanol by vortexing for $2 \mathrm{~min}$ followed by ultrasonic extraction in ice-cold water for $10 \mathrm{~min}$. The extracts were purified using $2 \mathrm{~mL}$ cold chloroform and $0.8 \mathrm{~mL}$ cold water. The resulting upper layer was dried, and redissolved in $1 \mathrm{~mL}$ water. The resulting solution was mixed with $30 \mathrm{mg}$ PVPP, stood for $60 \mathrm{~min}$, and centrifuged $\left(10,000 \times g, 4{ }^{\circ} \mathrm{C}, 10 \mathrm{~min}\right)$. The final supernatant was loaded to an Amberlite XAD-2 column ( $1 \mathrm{~mL}$ ) and eluted with $5 \mathrm{~mL}$ water (to remove sugars etc.), $5 \mathrm{~mL}$ pentane:dichloromethane (2/1) (to remove free aroma compounds), and $5 \mathrm{~mL}$ methanol. The methanol eluent was dried using nitrogen stream, and redissolved in $800 \mu \mathrm{L}$ of $50 \mathrm{mM}$ citric acid buffer ( $\mathrm{pH}$ 6.0) containing PD and $\beta$-glucosidase, and reacted at $37{ }^{\circ} \mathrm{C}$ for $16 \mathrm{~h}$. Afterwards, $144 \mathrm{mg}$ of sodium chloride was added to the reaction solution, and stood for $15 \mathrm{~min}$ followed by adding $0.5 \mathrm{nmol}$ of 
ethyl decanoate as an internal standard. The solution was extracted by $800 \mu \mathrm{L}$ extraction mixture (hexane:ethyl acetate, 1/1). After centrifugation, the extraction solution was collected, dried using anhydrous sodium sulfate, concentrated to $100 \mu \mathrm{L}$ under a stream of nitrogen, and followed by GC-MS analysis. The GC-MS condition and quantitative analysis of aroma compounds from glycosidic hydrolysis were the same as those described above.

\subsection{Transcript Expression Analysis of Key Genes Involved in Formation of Tea Aroma Compounds}

Total RNA was obtained using Quick RNA isolation Kit (Huayueyang Biotechnology Co., Ltd., Beijing, China). The cDNA was reversely transcribed from total RNA using PrimeScript RT Reagent Kit with gDNA Eraser (Takara Bio Inc., Kyoto, Japan) according to the manufacturer's instruction. Gene transcript expression was determined by quantitative real time PCR (qRT-PCR). The reaction was performed in a $0.2 \mathrm{~mL}$ microtube containing iTaq $^{\mathrm{TM}}$ Universal SYBR ${ }^{\circledR}$ Green Supermix $(10 \mu \mathrm{L})$ (Bio-Rad, Hercules, CA, USA), $0.2 \mu \mathrm{M}$ of each specific primer, 20-fold diluted cDNA (2 $\mu \mathrm{L})$, and ddH $\mathrm{H}_{2} \mathrm{O}$ $(6 \mu \mathrm{L})$. The encoding elongation factor1 (CsEF1) was used as an internal reference gene. The related genes specific primers of qRT-PCR are shown in Table 1. The qRT-PCR was carried out on Roche LightCycle 480 (Roche Applied Science, Mannheim, Germany) under condition of one cycle of $95^{\circ} \mathrm{C}$ for $60 \mathrm{~s}, 40$ cycles of $95{ }^{\circ} \mathrm{C}$ for $15 \mathrm{~s}$, and $60{ }^{\circ} \mathrm{C}$ for $30 \mathrm{~s}$. A melt curve was performed at the end of each reaction to verify PCR product specificity. The $2^{-\Delta \Delta c t}$ method was used to calculate the relative expression level. Changes in mRNA levels of related genes were normalized to that of CsEF1.

Table 1. The primers used for quantitative real time PCR (qRT-PCR) in the study.

\begin{tabular}{|c|c|c|c|}
\hline Gene & Accession Number & Forward Primer $5^{\prime}-3^{\prime}$ & Reverse Primer $5^{\prime}-3^{\prime}$ \\
\hline CsEF1 & KA280301.1 & TTGGACAAGCTCAAGGCTGAACG & ATGGCCAGGAGCATCAATGACAGT \\
\hline$C s P D$ & AB088027.1 & CCAAAGGTTCGGAATTGTCTATG & GCGCTTTTAGTCATACACCGA \\
\hline CsGT1 & AB847092 & TGAAGAAGGAAGCAGAAGAAGC & GGCTCATGATTCAACCGG \\
\hline CsGT2 & AB847093 & GAGGACATAAGGATTAAAGCGAG & TTTTCAACCCACTTAAATATTTCAATA \\
\hline CsLIS1 & KF006849 & ACATTGCAAGGATGGTTCC & ATGAGCATTACAGGTGCTAGCT \\
\hline CsLIS2 & KY033151 & GTCAATGTTCCGTGATACTGTTTC & ACACCAAGATAGACACCСТACTTTC \\
\hline CsLIS/NES-1 & KF006849 & TCCAACСССТСAATACAGAAAGACTATC & TTGGCTTTGTAGAAGTGCTTCAATCTC \\
\hline CsLIS/NES-2 & - & GAATGACAATCCAGGCATTG & TGGTGAGAATGGATTTGGAG \\
\hline
\end{tabular}

EF1, encoding elongation factor $1 ; P D, \beta$-primeverosidase; GT, glycosyltransferase; LIS, linalool synthase; LIS/NES, linalool synthase/(E)-nerolidolsynthase.

\subsection{Extraction and Analysis of GDP in Tea Leaves}

The method used was taken from our previous studies with some modifications [23,29]. Finely

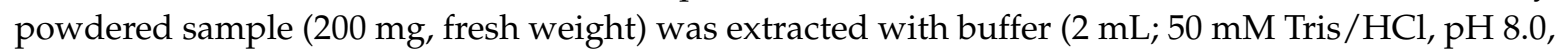
$20 \mathrm{mM}$ DTT, $20 \mathrm{mM} \mathrm{MgCl}_{2}$, and 5\% glycerin) containing $10 \mathrm{mM}$ sodium molybdate as phosphatase inhibitor by vortexing for $2 \mathrm{~min}$, followed by ultrasonic extraction in ice-cold water for $10 \mathrm{~min}$, and centrifugation $\left(10,000 \times g, 4{ }^{\circ} \mathrm{C}, 10 \mathrm{~min}\right)$. The supernatant $(1.6 \mathrm{~mL})$ was extracted with hexane $(1.6 \mathrm{~mL})$ five times to remove free aromas. The above buffer $(800 \mu \mathrm{L})$ was added and the aqueous fraction was incubated at $37^{\circ} \mathrm{C}$ for $30 \mathrm{~min}$ with $1 \mathrm{M} \mathrm{H}_{2} \mathrm{SO}_{4}(2 \mathrm{~mL})$. The reaction solution was cooled down for $3 \mathrm{~min}$ on ice and then the reaction was stopped by adding $100 \mu \mathrm{L}$ of $4 \mathrm{M} \mathrm{NaOH}$. The resultant mixture was extracted with $1 \mathrm{~mL}$ of hexane containing $0.5 \mathrm{nmol}$ of ethyl decanoate as the internal standard. The extract was then subjected to GC-MS analysis as described above. The GDP content was calculated based on standard curves which were constructed by plotting the concentration of linalool against the peak area of the authentic standard.

\subsection{Statistical Analysis}

Statistical analysis was performed using SPSS software, version 18.0 (SPSS Inc., Chicago, IL, USA). Two-tailed student's $t$ test was used to determine the differences between the normal and albino tea leaves. 


\section{Conclusions}

Herein, we detected the differential accumulation of aroma metabolites (aroma compounds) in albino-induced yellow tea leaves and normal green leaves. The albino-induced yellow tea leaves had lower amounts of free tea aroma compounds than the normal green tea leaves. In addition, most glycosidically bound aroma compounds in albino-induced yellow leaves were lower than those in normal green leaves. The lower content of linalool in albino-induced yellow tea leaves was due to lower GDP content compared with normal green tea leaves. This information will help us realize profiles of tea aromas and their formation in albino tea leaves. Furthermore, the results obtained in this study will help us to precisely evaluate the advantages (high amino acids) and disadvantages (low aromas) of albino-induced yellow tea leaves used to make tea products.

Supplementary Materials: The following are available online, Figure S1: GC-MS TIC chromatograms of endogenous free aroma compounds in normal green and albino-induced yellow tea leaves collected in April, August, and December, respectively; Table S1: Identified aroma compounds in the study.

Author Contributions: Z.Y. (Ziyin Yang) conceived the experiments. F.D., L.Z, Z.Y. (Zhenming Yu), J.L., J.T., and X.S. conducted the experiments. Z.Y. (Ziyin Yang), F.D., and L.Z. analysed the results. Z.Y. (Ziyin Yang), F.D., and L.Z. wrote the manuscript. All authors reviewed the manuscript.

Funding: This study was supported by the financial support from the Guangdong Province Higher Vocational Colleges \& Schools Pearl River Scholar, the Foundation of Science and Technology Program of Guangzhou [201804010097], the Guangdong Science and Technology Program [2017A020208015], the Guangdong Natural Science Foundation [2016A030313652, 2016A030306039, and 2018A030313457], and the Guangdong Innovation Team of Modern Agricultural Industry Technology System [2018LM1143].

Conflicts of Interest: The authors declare no conflict of interest.

\section{Abbreviations}

The following abbreviations are used in this manuscript:

$\begin{array}{ll}\text { VFADs } & \text { Aroma fatty acid derivatives } \\ \text { VTs } & \text { Aroma terpenes } \\ \text { VPBs } & \text { Aroma phenylpropanoids/benzenoids } \\ \text { PVPP } & \text { Polyvinylpolypyrrolidone } \\ \text { GDP } & \text { Geranyl diphosphate } \\ \text { RI } & \text { Retention indices } \\ \text { GBVs } & \text { Glycosidically bound aromas } \\ \text { EF1 } & \text { Encoding elongation factor 1 } \\ \text { PD } & \text { 3-Primeverosidase } \\ \text { GT } & \text { Glycosyltransferase } \\ \text { LIS } & \text { Linalool synthase } \\ \text { LIS/NES } & \text { Linalool synthase/(E)-nerolidol synthase }\end{array}$

\section{References}

1. Bushman, J.L. Green tea and cancer in humans: A review of the literature. Nutr. Cancer 1998, 31, 151-159. [CrossRef] [PubMed]

2. Trevisanato, S.I. Tea and health. Nutr. Rev. 2000, 58, 1-10. [CrossRef] [PubMed]

3. Wan, X.C. Tea Biochemistry, 3rd ed.; China Agriculture Press: Beijing, China, 2003. (In Chinese)

4. Yang, Z.Y.; Baldermann, S.; Watanabe, N. Recent studies of the aroma compounds in tea. Food Res. Int. 2013, 53, 585-599. [CrossRef]

5. Zeng, L.T.; Watanabe, N.; Yang, Z.Y. Understanding the biosyntheses and stress response mechanisms of aroma compounds in tea (Camellia sinensis) to safely and effectively improve tea aroma. Crit. Rev. Food Sci. 2018. [CrossRef] [PubMed]

6. Wang, K.R.; Li, M.; Zhang, L.J.; Liang, Y.R. Studies on classification of albino tearesources. J. Tea 2015, 41, 126-129. (In Chinese) 
7. Wang, W.; Guo, Y.L. Development and application of albino tea varieties. J. Food Saf. Qual. 2017, 8, 3104-3110. (In Chinese)

8. Du, Y.Y.; Chen, H.; Zhong, W.L.; Wu, L.Y.; Ye, J.H.; Lin, C.; Zheng, X.Q.; Lu, J.L.; Liang, Y.R. Effect of temperature on accumulation of chlorophylls and leaf ultrastructure of low temperature induced albino tea plant. Afr. J. Biotechnol. 2008, 7, 1881-1885. [CrossRef]

9. Du, Y.Y.; Shin, S.; Wang, K.R.; Lu, J.L.; Liang, Y.R. Effect of temperature on the expression of genes related to the accumulation of chlorophylls and carotenoids in albino tea. J. Hortic. Sci.Biotechnol. 2009, 84, 365-369. [CrossRef]

10. Wang, K.K.; Li, N.N.; Du, Y.Y.; Liang, Y.R. Effect of sunlight shielding on leaf structure and amino acids concentration of light sensitive albino tea plan. Afr. J. Biotechnol. 2013, 12, 5535-5539.

11. Song, L.; Ma, Q.; Zou, Z.; Sun, K.; Yao, Y.; Tao, J.; Kaleri, N.A.; Li, X. Molecular link between leaf coloration and gene expression of flavonoid and carotenoid biosynthesis in Camellia sinensis cultivar 'Huangjinya'. Front. Plant Sci. 2017, 8. [CrossRef] [PubMed]

12. Liu, G.F.; Han, Z.X.; Feng, L.; Gao, L.P.; Gao, M.J.; Gruber, M.Y.; Zhang, Z.L.; Xia, T.; Wan, X.C.; Wei, S. Metabolic flux redirection and transcriptomic reprogramming in the albino tea cultivar 'Yu-Jin-Xiang' with an emphasis on catechin production. Sci. Rep. 2017, 7, 45062. [CrossRef] [PubMed]

13. Cheng, S.H.; Fu, X.M.; Liao, Y.Y.; Xu, X.L.; Zeng, L.T.; Tang, J.C.; Li, J.L.; Lai, J.H.; Yang, Z.Y. Differential accumulation of specialized metabolite L-theanine in green and albino-induced yellow tea (Camellia sinensis) leaves. Food Chem. 2019, 276, 93-100. [CrossRef]

14. Li, Q.; Huang, J.A.; Liu, S.Q.; Li, J.; Yang, X.H.; Liu, Y.S.; Liu, Z.H. Proteomic analysis of young leaves at three developmental stages in an albino tea cultivar. Proteome Sci. 2011, 9, 44. [CrossRef] [PubMed]

15. Cheng, S.H.; Fu, X.M.; Wang, X.Q.; Liao, Y.Y.; Zeng, L.T.; Dong, F.; Yang, Z.Y. Studies on the biochemical formation pathway of the amino acid L-theanine in tea (Camellia sinensis) and other plants. J. Agric. Food Chem. 2017, 65, 7210-7216. [CrossRef] [PubMed]

16. Yang, Z.Y.; Kobayashi, E.; Katsuno, T.; Asanuma, T.; Fujimori, T.; Ishikawa, T.; Tomomura, M.; Mochizuki, K.; Watase, T.; Nakamura, Y.; et al. Characterisation of volatile and non-volatile metabolites in etiolated leaves of tea (Camellia sinensis) plants in the dark. Food Chem. 2012, 135, 2268-2276. [CrossRef] [PubMed]

17. Chen, Y.Y.; Fu, X.M.; Mei, X.; Zhou, Y.; Cheng, S.H.; Zeng, L.T.; Dong, F.; Yang, Z.Y. Proteolysis of chloroplast proteins is responsible for accumulation of amino acids in dark-treated tea (Camellia sinensis) leaves. J. Proteom. 2017, 157, 10-17. [CrossRef] [PubMed]

18. Mizutani, M.; Nakanishi, H.; Ema, J.I.; Ma, S.J.; Noguchi, E.; Inohara-Ochiai, M.; Fukuchi-Mizutani, M.; Nakao, M.; Sakata, K. Cloning of $\beta$-primeverosidase from tea leaves, a key enzyme in tea aroma formation. Plant Physiol. 2002, 130, 2164-2176. [CrossRef] [PubMed]

19. Zhou, Y.; Dong, F.; Kunimasa, A.; Zhang, Y.Q.; Cheng, S.H.; Lu, J.M.; Zhang, L.; Murata, A.; Mayer, F.; Fleischmann, P.; et al. Occurrence of glycosidically conjugated 1-phenylethanol and its hydrolase $\beta$-primeverosidase in tea (Camellia sinensis) flowers. J. Agr. Food Chem. 2014, 62, 8042-8050. [CrossRef] [PubMed]

20. Zhou, Y.; Zeng, L.T.; Gui, J.D.; Liao, Y.Y.; Li, J.L.; Tang, J.C.; Meng, Q.; Dong, F.; Yang, Z.Y. Functional characterizations of $\beta$-glucosidases involved in aroma compound formation in tea (Camellia sinensis). Food Res. Int. 2017, 96, 206-214. [CrossRef] [PubMed]

21. Ohgami, S.; Ono, E.; Horikawa, M.; Murata, J.; Toyonaga, H.; Ohba, Y.; Dohra, H.; Asai, T.; Mizutani, M.; Watanabe, N.; et al. Aroma glycosylation in tea plants: sequential glycosylationsfor the biosynthesis of aroma $\beta$-primeverosides are catalyzed by two Camellia sinensis glycosyltransferases. Plant Physiol. 2015, 168, 464-477. [CrossRef] [PubMed]

22. Gui, J.D.; Fu, X.M.; Zhou, Y.; Katsuno, T.; Mei, X.; Deng, R.F.; Xu, X.L.; Zhang, L.Y.; Dong, F.; Watanabe, N.; et al. Does enzymatic hydrolysis of glycosidically bound aroma compounds really contribute to the formation of aroma compounds during the oolong tea manufacturing process? J. Agr. Food Chem. 2015, 63, 6905-6914. [CrossRef] [PubMed]

23. Fu, X.M.; Chen, Y.Y.; Mei, X.; Katsuno, T.; Kobayashi, E.; Dong, F.; Watanabe, N.; Yang, Z.Y. Regulation of formation of aroma compounds of tea (Camellia sinensis) leaves by single light wavelength. Sci. Rep. 2015, 5, 16858. [CrossRef] [PubMed] 
24. Zhou, Y.; Zeng, L.T.; Liu, X.Y.; Gui, J.D.; Mei, X.; Fu, X.M.; Dong, F.; Tang, J.C.; Zhang, L.Y.; Yang, Z.Y. Formation of (E)-nerolidol in tea (Camellia sinensis) leaves exposed to multiple stresses during tea manufacturing. Food Chem. 2017, 231, 78-86. [CrossRef] [PubMed]

25. Mei, X.; Liu, X.Y.; Zhou, Y.; Wang, X.Q.; Zeng, L.T.; Fu, X.M.; Li, J.L.; Tang, J.C.; Dong, F.; Yang, Z.Y. Formation and emission of linalool in tea (Camellia sinensis) leaves infested by tea green leafhopper (Empoasca (Matsumurasca) onukii Matsuda). Food Chem. 2017, 237, 356-363. [CrossRef] [PubMed]

26. Liu, G.F.; Liu, J.J.; He, Z.R.; Wang, F.M.; Yang, H.; Yan, Y.F.; Gao, M.J.; Gruber, M.Y.; Wan, X.C.; Wei, S. Implementation of CsLIS/NES in linalool biosynthesis involves transcript splicing regulation in Camellia sinensis. Plant Cell Environ. 2018, 41, 176-186. [CrossRef] [PubMed]

27. Zeng, L.T.; Zhou, Y.; Fu, X.M.; Liao, Y.Y.; Yuan, Y.F.; Jia, Y.X.; Dong, F.; Yang, Z.Y. Biosynthesis of jasmine lactone in tea (Camellia sinensis) leaves and its formation in response to multiple stresses. J. Agric. Food Chem. 2018, 66, 3899-3909. [CrossRef] [PubMed]

28. Wang, X.Q.; Zeng, L.T.; Liao, Y.Y.; Zhou, Y.; Xu, X.L.; Dong, F.; Yang, Z.Y. An alternative pathway for the formation of aromatic aroma compounds derived from L-phenylalanine via phenylpyruvic acid in tea (Camellia sinensis (L.) O. Kuntze) leaves. Food Chem. 2019, 270, 17-24. [CrossRef] [PubMed]

29. Zeng, L.T.; Zhou, Y.; Fu, X.M.; Mei, X.; Cheng, S.H.; Gui, J.D.; Dong, F.; Tang, J.C.; Ma, S.Z.; Yang, Z.Y. Does oolong tea (Camellia sinensis) made from a combination of leaf and stem smell more aromatic than leaf-only tea? Contribution of the stem to oolong tea aroma. Food Chem. 2017, 237, 488-498. [CrossRef] [PubMed]

30. Zhu, Y.; Lv, H.P.; Shao, C.Y.; Kang, S.; Zhang, Y.; Guo, L.; Dai, W.D.; Tan, J.F.; Peng, Q.H.; Lin, Z. Identification of key odorants responsible for chestnut-like aroma quality of green teas. Food Res. Int. 2018, 108, 74-82. [CrossRef] [PubMed]

Sample Availability: Samples of the compounds n-Alkanes (C8-C40), benzaldehyde, benzyl alcohol, (Z)-3-hexenyl acetate, methyl salicylate, 2-phenylethanol, ethyl n-decanoate, geraniol, 1-hexanol, (Z)-3-hexenol, phenylacetaldehyde, $\alpha$-farnesene, $(E)$-nerolidol, linalool, and linalool oxide are available from the authors.

(c) 2018 by the authors. Licensee MDPI, Basel, Switzerland. This article is an open access article distributed under the terms and conditions of the Creative Commons Attribution (CC BY) license (http://creativecommons.org/licenses/by/4.0/). 